\title{
Reversal of severe methotrexate-induced intestinal damage using enteral $n-3$ fatty acids
}

\author{
Tal Koppelmann ${ }^{1,2}$, Yulia Pollak ${ }^{1}$, Jorge Mogilner ${ }^{3}$, Jacob Bejar ${ }^{4}$, Arnold G. Coran ${ }^{5}$ \\ and Igor Sukhotnik ${ }^{1,3 *}$ \\ ${ }^{1}$ Laboratory of Intestinal Adaptation and Recovery, The Bruce Rappaport Faculty of Medicine, Technion-Israel Institute of \\ Technology, Bnai Zion Medical Center, Haifa, Israel \\ ${ }^{2}$ Department of Surgery, Bnai Zion Medical Center, Haifa, Israel \\ ${ }^{3}$ Department of Pediatric Surgery, Bnai Zion Medical Center, 47 Golomb Street, PO Box 4940, Haifa 31048, Israel \\ ${ }^{4}$ Department of Pathology, Bnai Zion Medical Center, Haifa, Israel \\ ${ }^{5}$ Section of Pediatric Surgery, C.S. Mott Children's Hospital, University of Michigan Medical School, Ann Arbor, MI, USA \\ (Submitted 10 October 2011 - Final revision received 16 January 2012 - Accepted 26 January 2012 - First published online 28 March 2012)
}

\section{Abstract}

Growing evidence suggests that $n-3$ PUFA and their specific lipid mediators can reduce the activity of inflammatory processes. In the present study, we evaluated the effects of oral $n$-3 PUFA supplementation on intestinal structural changes, enterocyte proliferation and apoptosis during methotrexate (MTX)-induced intestinal damage in the rat. A total of thirty-two male rats were divided into four experimental groups: control (CONTR) rats; CONTR- $n-3$ PUFA rats treated with oral administration of $n-3$ PUFA at a dose of $300 \mu \mathrm{g} / \mathrm{kg}$ once per $\mathrm{d} 72 \mathrm{~h}$ before and $72 \mathrm{~h}$ following vehicle injection; MTX rats treated with a single dose of MTX; MTX- $n-3$ PUFA rats treated with oral $n-3$ PUFA following the injection of MTX. Intestinal mucosal damage, mucosal structural changes, enterocyte proliferation and enterocyte apoptosis determined $72 \mathrm{~h}$ following MTX injection. Real-time PCR was used to determine B-cell lymphoma 2 (Bcl2)-associated X protein (Bax) and Bcl2 mRNA expression. Western blotting was used to determine phosphorylated extracellular signal-related kinase, $\beta$-catenin, Bax and Bcl2 protein levels. MTX- $n$-3 PUFA rats demonstrated a greater jejunal and ileal bowel weight, greater ileal mucosal weight, greater ileal mucosal DNA and protein levels, greater villus height in the jejunum and ileum and crypt depth in the ileum, compared with MTX animals. A significant decrease in enterocyte apoptosis in the ileum of MTX- $n-3$ PUFA rats ( $v$. MTX) was accompanied by decreased Bax mRNA and protein expression and increased $B c l 2$ mRNA levels. Thus, the treatment with oral $n-3$ PUFA prevented mucosal injury and improved intestinal recovery following MTX-injury in rats.

Key words: Methotrexate: Mucositis: Intestine: $\boldsymbol{n}$-3 PUFA: Rats

Over the past decades, growing evidence from several clinical and experimental studies has indicated that $n$ - 3 PUFA (DHA $(22: 6)+$ EPA $(20: 5))$ can not only reduce the activity of inflammatory processes but may also improve the immune system and protect the organism against the negative effect of the systemic inflammatory response syndrome ${ }^{(1)}$. The biologically active essential fatty acids such as arachidonic acid ( $n-6$ family) and EPA/DHA ( $n-3$ family) play an important role in inflammatory processes as eicosanoid and prostanoid precursors. Arachidonic acid is considered pro-inflammatory and prothrombotic, while EPA/DHA are usually labelled as anti-inflammatory and anti-thrombotic ${ }^{(2)}$. Recent evidence suggests that converting cellular membranes to an $n-6: n-3$ ratio close to 1:1 decreases the substrate availability necessary for the production of inflammatory cytokines and replaces them with anti-inflammatory mediators ${ }^{(3)}$. In addition, $n-3$ PUFA reduce T-cell receptor expression, phytomitogen response, antigenic stimulation responses by lymphocytes, IgA expression, delayed-type hypersensitivity responses and accessory cell function ${ }^{(4)}$. Dietary $n-3$ PUFA also decrease the production of different pro-inflammatory mediators such as IL- 1, IL-2, IL- 6 and TNF- $\alpha^{(5)}$. The positive anti-inflammatory effect of $n$-3 PUFA has been described in several inflammatory conditions such as inflammatory bowel disease ${ }^{(6,7)}$, rheumatoid arthritis ${ }^{(8,9)}$ and hepatitis ${ }^{(10)}$.

Oral and gastrointestinal mucositis are frequent complications of chemotherapy, in particular with drugs affecting DNA synthesis (such as fluorouracil, methotrexate and cytarabine), contributing not only to the morbidity of treatment but its cost as well ${ }^{(11,12)}$. Mucositis limits the patient's

Abbreviations: BrdU, bromodeoxyuridine; ERK, extracellular signal-related kinase; MAPK, mitogen-activated protein kinase.

*Corresponding author: Dr I. Sukhotnik, fax +97248359620, email igor-dr@internet-zahav.net 
ability to tolerate chemotherapy or radiation therapy, prolongs hospital stay, increases re-admission rates, compromises the patient's nutritional status, affects the patient's quality of life and is occasionally fatal. Severe inflammation of the intestinal mucosa plays a significant role in the development of chemotherapy-induced mucositis and is a major characteristic of the condition ${ }^{(13)}$. Different inflammatory mediators and cytokines, such as leukotriene B4 and PGE2, act to amplify signalling cascades, induce apoptosis and cause further tissue damage. Since reduction of inflammation could truncate the time course of the condition, we hypothesised that $n-3$ PUFA might reverse chemotherapy-induced intestinal damage and/or exert beneficial effects on downstream phases associated with repair and healing.

\section{Materials and methods}

\section{Animals}

This experiment and animal care were conducted in compliance with the guidelines established by the 'Guide for the Care and Use of Laboratory Animals', Rappaport Faculty of Medicine, Technion (Haifa, Israel). Male Sprague-Dawley rats (250-300 g) were used in the present study. The animals were housed in wire-bottomed cages and were acclimatised at $21^{\circ} \mathrm{C}$ on a $12 \mathrm{~h}$ light $-12 \mathrm{~h}$ dark cycle for a minimum of $3 \mathrm{~d}$ before the experiment. The rats were allowed access to water and chow ad libitum.

\section{Experimental design}

A total of thirty-two rats were divided randomly into four experimental groups of eight rats each. (1) Group A - control (CONTR) rats underwent intraperitoneal injection of vehicle; (2) group B (CONTR- $n$-3 PUFA) animals were treated with oral administration of $n$ - 3 PUFA once per $\mathrm{d}$ at a dose of $300 \mu \mathrm{g} / \mathrm{kg}$ per d (EPA $180 \mu \mathrm{g}+$ DHA $120 \mu \mathrm{g}$ ) for $48 \mathrm{~h}$ followed by vehicle injection followed by $72 \mathrm{~h}$ of additional PUFA dietary treatment; (3) group C rats (MTX) were treated with a single intraperitoneal injection of MTX $(20 \mathrm{mg} / \mathrm{kg}$ ); (4) group D (MTX- $n$-3 PUFA) animals were pre-treated with oral $n$-3 PUFA given similarly to group $\mathrm{B}, 48 \mathrm{~h}$ before and $72 \mathrm{~h}$ after MTX injection.

\section{Intestinal mucosal parameters}

All animals were killed $72 \mathrm{~h}$ following MTX or vehicle injection. The entire small intestine was carefully removed and placed on ice. Portions of the intestine $10 \mathrm{~cm}$ distal to the ligament of Treitz and $10 \mathrm{~cm}$ proximal to the ileo-caecal region were removed, representing the proximal (jejunum) and distal (ileum) segments. Intestinal segments were washed with cold saline, dried and weighed. Each segment was rinsed thoroughly with physiological saline and opened longitudinally on its antimesenteric border to expose the intestinal mucosa. The mucosa was scraped from the underlying tissue with a glass slide. Total RNA, DNA and protein from the jejunum and ileum were extracted sequentially with a TRIzol reagent.

\section{Histological examination}

Tissue samples were removed from the jejunum and ileum and were immediately fixed in $4 \%$ neutral-buffered formalin. The samples were then embedded in paraffin and sectioned. Deparaffinised $5 \mu \mathrm{m}$ sections were stained with haematoxylin and eosin. The villus height and crypt depth for each specimen were measured using an objective mounted micrometer $(100 \times$ magnification) and an optical microscope $(10 \times 100$ magnification $)$. Villus height and crypt depth data were from eight rats, and each measurement consisted of the mean of five villi and crypts.

The mucosal damage of the small bowel was graded using an intestinal injury score as described by Kesik et al. ${ }^{(14)}$ The following parameters were investigated in the jejunum and ileum: (1) degeneration of surface and crypt epithelium; (2) degeneration of the villus structure, vacuolisation in the surface epithelium; (3) inflammatory cell infiltration, and bleeding and oedema in the lamina propria. For each parameter, a score was given using a semi-quantitative scale as follows: $0=$ none, $1=$ mild, $2=$ moderate, $3=$ severe, giving a maximum possible score of 9 for each intestinal region.

\section{Enterocyte proliferation and apoptosis}

Crypt cell proliferation was assessed using 5-bromodeoxyuridine. Standard bromodeoxyuridine (BrdU) labelling reagent (Zymed Laboratories, Inc.) was injected intraperitoneally at a concentration of $1 \mathrm{ml} / 100 \mathrm{~g}$ body weight $2 \mathrm{~h}$ before killing. Tissue slices $(5 \mu \mathrm{m})$ were stained with a biotinylated monoclonal anti-BrdU antibody system provided in a kit form (Zymed Laboratories, Inc.). An index of proliferation was determined as the ratio of crypt cells staining positively for BrdU per ten crypts.

Additional $5 \mu \mathrm{m}$-thick sections were prepared to establish the degree of enterocyte apoptosis. Immunohistochemistry for active caspase-3 (caspase-3 cleaved concentrated polyclonal antibody; dilution 1:100; Biocare Medical) was performed for identification of apoptotic cells using a combination of the streptavidin-biotin-peroxidase method and microwave antigen retrieval on formalin-fixed, paraffin-embedded tissues according to the manufacturer's protocols. The apoptotic index was defined as the number of apoptotic cells/1000 cells per ten villi.

A qualified pathologist blinded as to the source of intestinal tissue performed all measurements.

\section{Expression of $\mathrm{Bax}$ and $\mathrm{Bcl} 2$ genes (real-time $P(R)$}

Expression of Bax and Bcl2 levels was determined by quantitative real-time PCR (7500 Real-Time PCR System; Applied Biosystems) on complementary DNA samples using the Cyber Green Master Mix (ROVALAB) with the exception of template and primers. Primers for Rattus norvegicus Bax and $\mathrm{Bcl} 2$ were synthesised by Syntezza Bioscience Limited.

\section{Western blotting}

Tissue was homogenised in radioimmuno precipitation assay (RIPA) lysis buffer containing $50 \mathrm{~mm}$-Tris- $\mathrm{HCl}$ (pH 7.4), 
$150 \mathrm{~mm}-\mathrm{NaCl}, 1 \% \mathrm{NP}-40$ and 2 mm-EDTA, supplemented with a cocktail of protease and phosphatase inhibitors. Protein concentrations were determined by Bradford reagent according to the manufacturer's instructions. Samples containing equal amounts of total protein $(30 \mu \mathrm{g})$ were resolved by SDS-PAGE under reducing conditions. After electrophoresis, proteins were transferred to a PVDF membrane and probed with various primary antibodies to anti-Bcl-2 antibody (1:1000 dilution, sc-7382), anti-Bax antibody (1:200 dilution, sc-493), anti-phosphorylated extracellular signal-related kinase (ERK) antibody (1:2500 dilution, sc-7383), anti- $\beta$-catenin antibody (1:1000 dilution, sc-7199) and anti-ERK2 antibody (1:1000 dilution, sc-56899). Horseradish peroxidase-conjugated secondary antibody was purchased from Jackson ImmunoResearch Laboratories, Inc. and an enhanced chemiluminescent substrate from Biological Industries. The optical density of the specific protein bands was quantified by using a densitometer (Vilber Lourmat)

\section{Statistical analysis}

Data are expressed as means with their standard errors. A oneway ANOVA for comparison, followed by Tukey's test for pairwise comparison, was used for statistical analysis. Prism software was used (GraphPad Software, Inc.) and statistical significance was defined as $P<0.05$.

\section{Results}

\section{Body-weight changes}

MTX rats (group C) demonstrated a significant decrease in final body weight (99 (SEM 3) $v$. 107 (SEM 2)\% initial, $P<0.05$ ) compared with the control animals (group A) (Fig. 1). Although treatment with PUFA in both control and MTX animals resulted in a trend towards an increase in final body weight $(P=0.13$ and $P=0.095$, correspondingly), this trend was not statistically significant.

\section{Intestinal damage score}

Histologically, MTX animals exhibited a significant loss of crypt architecture and signs of crypt remodelling, severe villous epithelial atrophy, degeneration and shortening of the villus length, and polymorphonuclear leucocyte infiltration in the lamina propria. Histological damage was initially assessed in the jejunum and ileum by the semi-quantitative score, which included three criteria that were markedly affected by methotrexate. Administration of $n$ - 3 PUFA in the control animals (group B) had no significant effect on the semi-quantitative damage score compared with the control rats (group A) (Fig. 2). Intestinal damage as a result of methotrexate administration was greatest in the distal ileum (7.7 (sem $0 \cdot 2) v \cdot 0 \cdot 1$ (SEM 0.01), $P<0 \cdot 001)$ and was less significant in the proximal jejunum $(3 \cdot 2$ (SEM 0.2) $v$. $0 \cdot 1$ (SEM 0.01), $P<0 \cdot 001)$. Treatment with oral $n-3$ PUFA of MTX rats (group D) resulted in a significant decrease in intestinal damage score in the proximal jejunum (1.9 (SEM 0.09) v. $3 \cdot 2$

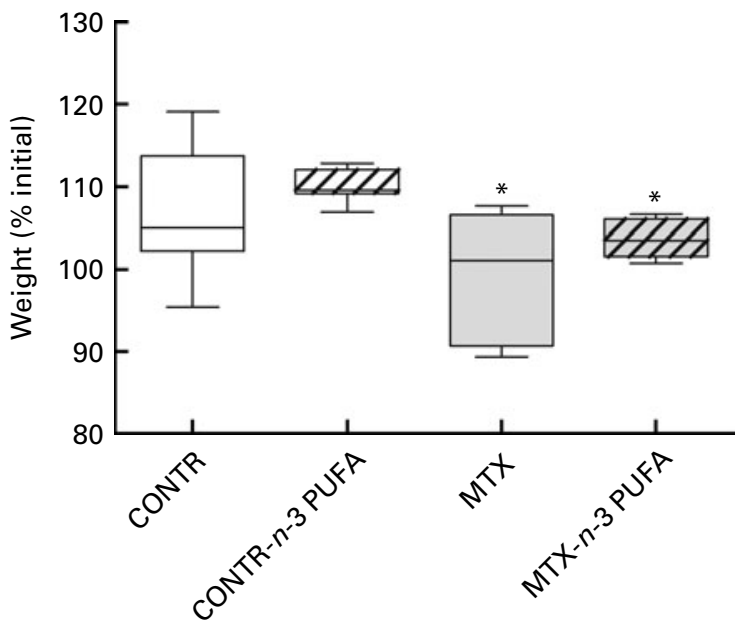

Fig. 1. Effects of methotrexate (MTX) and n-3 PUFA on body-weight changes. Values are means, with their standard errors represented by vertical bars. * Mean values were significantly different for MTX and MTX- $n-3$ PUFA rats from those of the control (CONTR) rats $(P<0.05)$.

(sem 0.2), $P<0.05$ ) and the distal ileum (2.5 (SEm 0.84) $v .7 \cdot 7$ (sEm 0.2), $P<0 \cdot 001$ ) compared with MTX animals (group C).

\section{Intestinal mucosal parameters}

Treatment with oral $n-3$ PUFA of the control animals (group B) did not change significantly intestinal mucosal parameters compared with the control animals (group A) (Figs. 3 and 4). MTX rats (group B) showed a significant decrease in bowel weight in the jejunum $(26 \%, P<0.001)$ and ileum $(19 \%$ increase, $P=0 \cdot 001$ ), mucosal weight in the jejunum (2-fold decrease, $P<0.001$ ) and ileum (2-fold decrease, $P<0.001$ ) (Fig. 3), mucosal DNA in the jejunum (3-fold decrease, $P=0.003)$ and ileum (2-fold decrease, $P=0.004)$, and mucosal protein in the jejunum (3-fold decrease, $P=0.009$ ) and ileum (2-fold decrease, $P=0.01$ ) (Fig. 4) compared with the control animals. Administration of $n$-3 PUFA (group D) resulted in a significant increase in jejunal ( $12 \%$ increase, $P<0.001)$ and ileal (23\% increase, $P<0.05)$ bowel weight, ileal (25\% increase, $P<0.05$ ) mucosal weight (Fig. 1), ileal mucosal DNA (3-fold increase, $P<0.01)$ and protein $(74 \%$ increase, $P<0.05)$ compared with MTX animals (group C).

\section{Microscopic bowel appearance}

CONTR- $n$-3 PUFA rats showed a similar microscopic appearance compared with the control animals. MTX rats exhibited a significant decrease in villus height in the jejunum (284 (SEM 18) v. 444 (SEM 29) $\mu \mathrm{m}, P<0.001)$ and ileum (223 (SEM 36) $v .371$ (SEM 26) $\mu \mathrm{m}, P<0 \cdot 001)$ as well as crypt depth in the jejunum (121 (SEM 9) $v .159$ (SEM 10) $\mu \mathrm{m}, P<0.001)$ and ileum (114 (SEM 14) v. 146 (SEM 8) $\mu \mathrm{m}, P<0.05)$ compared with the control animals (Fig. 5). n-3 PUFA-treated rats (group D) demonstrated a significant increase in jejunal (394 (SEM 57) v. 284 (SEM 18) $\mu \mathrm{m}, P<0 \cdot 05$ ) and ileal (316 (SEM 31) $v$. 223 (SEM 36) $\mu \mathrm{m}, P<0.05$ ) villus height as well as in jejunal (160 (sem 10) v. 121 (sem 9) $\mu \mathrm{m}, P<0 \cdot 01$ ) and ileal (149 (sEm 7) 

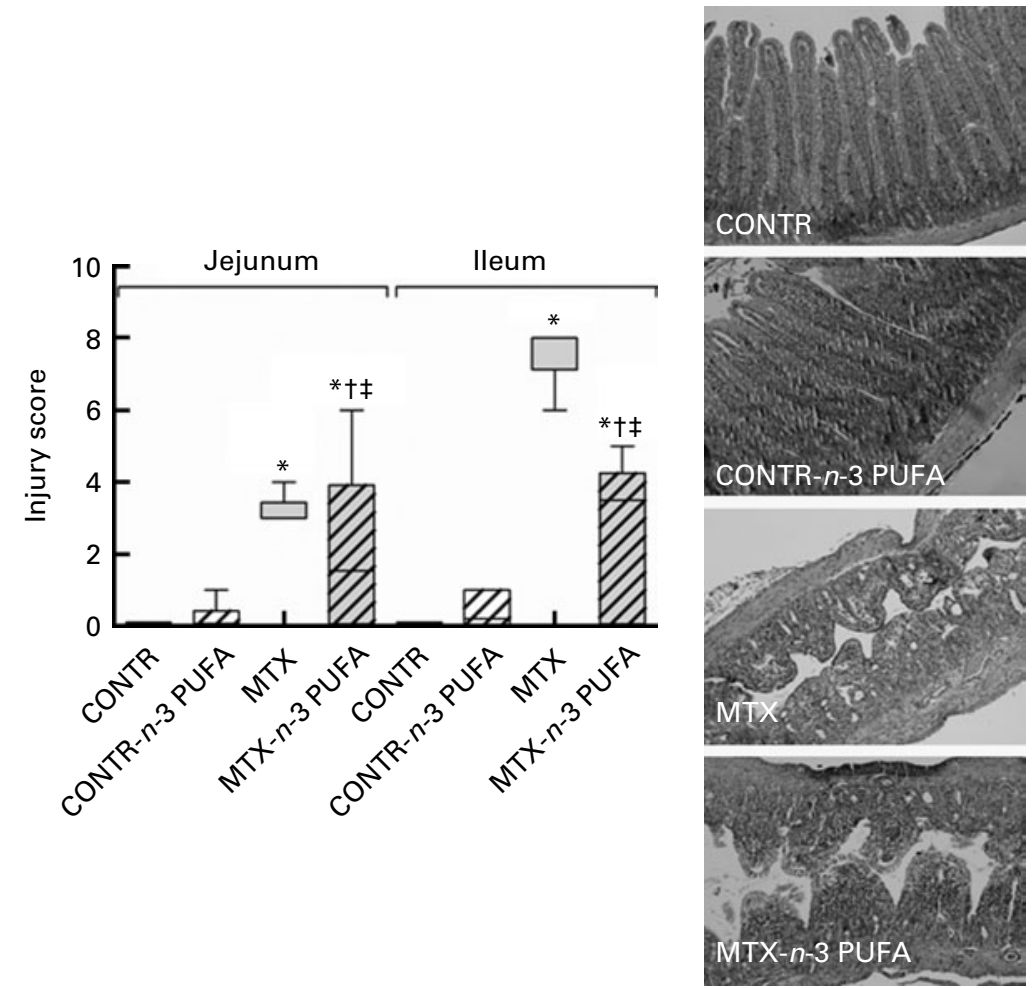

Fig. 2. Effects of methotrexate (MTX) and enteral $n-3$ PUFA on intestinal injury score. The following parameters were investigated: (1) degeneration of surface and crypt epithelium; (2) degeneration of the villus structure, vacuolisation in the surface epithelium; (3) inflammatory cell infiltration, and bleeding and oedema in the lamina propria. For each criterion, a score was given using a semi-quantitative scale as follows: $0=$ none, $1=$ mild, $2=$ moderate, $3=$ severe, giving a maximum possible score of 9 for each segment. Values are means, with their standard errors represented by vertical bars. ${ }^{*}$ Mean values were significantly different for MTX and MTX- $n-3$ PUFA rats from those of the control (CONTR) rats $(P<0.05)$. † Mean values were significantly different for MTX- $n$-3-PUFA rats from those of MTX rats $(P<0.05)$. $¥$ Mean values were significantly different for MTX and MTX- $n-3$ PUFA rats from those of CONTR- $n-3$ PUFA rats $(P<0.05)$.

v. 114 (SEM 14) $\mu \mathrm{m}, P<0.05$ ) crypt depth compared with MTX animals (group C).

\section{Enterocyte proliferation and apoptosis}

Treatment with oral $n$-3 PUFA of the control animals (group B) did not change significantly cell proliferation and apoptosis rates compared with the control animals (group A) (Fig. 6). MTX rats (group C) demonstrated a significant decrease in enterocyte proliferation index in the jejunum (125 (SEM 6) $v$. 177 (sem 9) BrdU-positive cells per ten crypts, $P<0 \cdot 001$ ) and ileum (120 (SEM 6) v. 185 (SEM 10) BrdU-positive cells per ten crypts, $P<0.001)$ compared with the control animals. Oral $n-3$ PUFA administration (group D) induced a significant increase in enterocyte proliferation index in the jejunum (179 (SEM 27) v. 125 (SEM 6) BrdU-positive cells per ten crypts, $P<0 \cdot 001$ ) and ileum (191 (sem 19) $v .120$ (sem 6) BrdU-positive cells per ten crypts, $P=0.02)$ compared with MTX animals (group C)

Significantly greater numbers of apoptotic cells appeared in the villus of the jejunum (2.5-fold increase, $P<0.05)$ and ileum (4-fold increase, $P<0 \cdot 001$ ) of MTX rats (group C) compared with the control animals (group A) (Fig. 6). Exposure to oral $n$-3 PUFA (group D) led to a significant decrease in apoptotic index in the jejunum (2.5-fold decrease, $P=0.03)$ and ileum (3-fold decrease, $P<0 \cdot 001)$ compared with MTX-untreated animals (group C).

\section{Expression of $\mathrm{Bax}$ and $\mathrm{Bcl} 2$ genes}

MTX animals demonstrated a significant 2-fold increase in Bax mRNA expression in the jejunum $(P<0.005)$ and ileum $(P<0.005)$ and a concomitant decrease in Bcl2 mRNA levels in the jejunum $(P<0.05)$ compared with the control animals (Fig. 7). Treatment of MTX rats with $n-3$ PUFA attenuated the pro-apoptotic effects of MTX. MTX- $n$ - 3 PUFA rats (group D) showed a significant decrease in Bax mRNA expression in the jejunum $(33 \%, P<0.05)$ and ileum $(42 \%, P<0.05)$ as well as a significant increase in $B c l 2$ mRNA expression in the ileum (2-fold increase, $P<0.05$ ) compared with MTX animals.

\section{Western blotting}

Treatment with oral $n-3$ PUFA of the control animals (group B) did not change significantly compared with the control animals (group A) proliferation- and apoptosis-related protein levels, which is in agreement with unchanged rates of cell proliferation and apoptosis. Decreased cell proliferation rates in MTX animals (group B) were accompanied by decreased levels of $\beta$-catenin (Fig. 8). In addition, increased cell apoptosis 


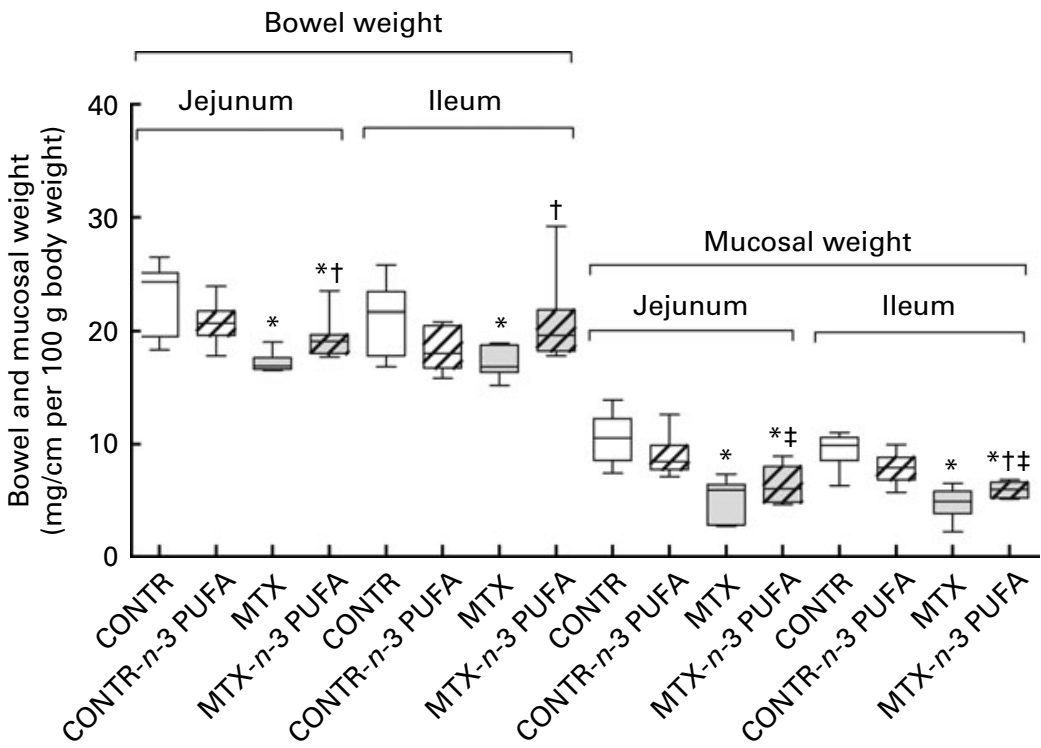

Fig. 3. Effects of methotrexate (MTX) and enteral $n$-3 PUFA on bowel and mucosal weight. Values are means, with their standard errors represented by vertical bars. * Mean values were significantly different for MTX and MTX- $n-3$ PUFA rats from those of the control (CONTR) rats $(P<0.05)$. $†$ Mean values were significantly different for MTX- $n$-3-PUFA rats from those of MTX rats $(P<0.05)$. $¥$ Mean values were significantly different for MTX and MTX- $n-3$ PUFA rats from those of CONTR- $n$-3 PUFA rats $(P<0.05)$.

was accompanied by increased Bax protein levels, which is in agreement with Bax mRNA expression (Fig. 7). Treatment with $n$-3 PUFA (group D) resulted in a significant increase in both phosphorylated ERK and $\beta$-catenin protein levels, which correlated with increased cell proliferation rates in this group compared with MTX-untreated animals (group B) (Fig. 8). MTX- $n-3$ PUFA animals demonstrated a significant decrease in Bax protein levels as well as an increase in $\mathrm{Bcl} 2$ protein levels compared with MTX animals. These findings correlate with decreased cell apoptosis in this group and are in agreement with the mRNA expressions.

\section{Discussion}

Since 1975, when it was reported that a high intake of marine fish containing $n$ - 3 fatty acids is associated with a low prevalence of coronary artery disease ${ }^{(15)}$, administration of fish oilbased nutritional formulas has evolved from a supportive to a therapeutic intervention in a number of critical care settings. Over the past 20 years, many studies and clinical investigations have demonstrated that $n$ - 3 PUFA are essential for normal growth and development and play an important role in the prevention and treatment of coronary artery disease,

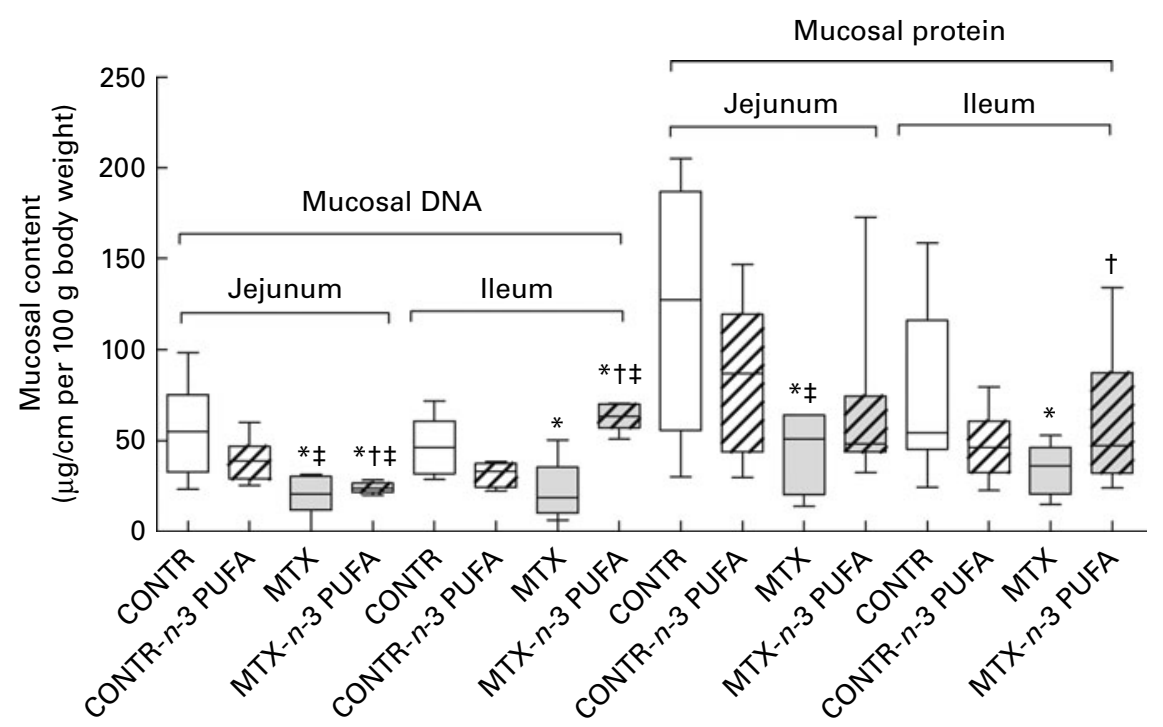

Fig. 4. Effects of enteral $n$-3 PUFA on mucosal DNA and protein content in a rat model of methotrexate (MTX)-induced mucositis. Values are means, with their standard errors represented by vertical bars. ${ }^{*}$ Mean values were significantly different for MTX and MTX- $n-3$ PUFA rats from those of the control (CONTR) rats $(P<0.05)$. † Mean values were significantly different for MTX- $n$-3-PUFA rats from those of MTX rats $(P<0.05)$. $\ddagger$ Mean values were significantly different for MTX and MTX- $n$-3 PUFA rats from those of CONTR- $n-3$ PUFA rats $(P<0.05)$. 


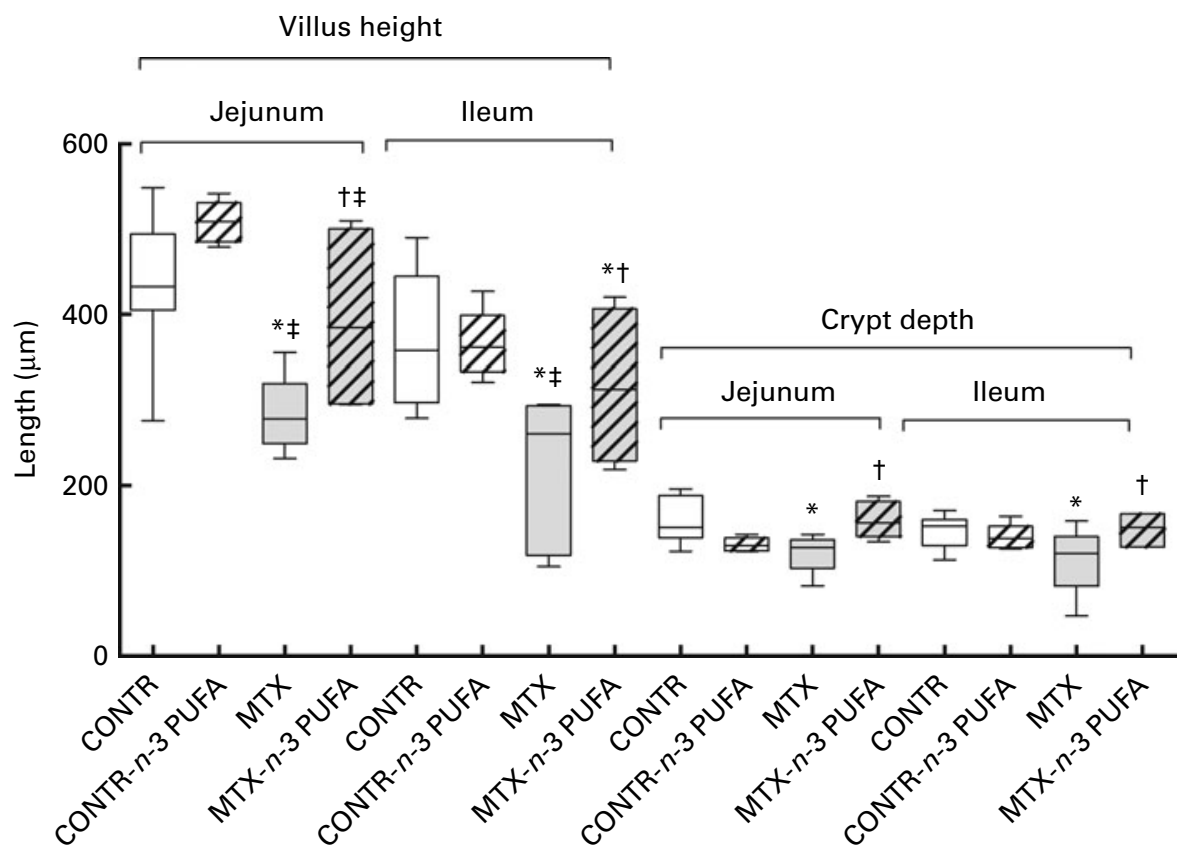

Fig. 5. Effects of methotrexate (MTX) and enteral $n$-3 PUFA on microscopic intestinal appearance. Values are means, with their standard errors represented by vertical bars. * Mean values were significantly different for MTX and MTX- $n-3$ PUFA rats from those of the control (CONTR) rats $(P<0.05)$. $†$ Mean values were significantly different for MTX-3-PUFA rats from those of MTX rats $(P<0.05)$. $¥$ Mean values were significantly different for MTX and MTX- $n-3$ PUFA rats from those of CONTR- $n$-3 PUFA rats $(P<0.05)$.

hypertension, diabetes, arthritis, other inflammatory and autoimmune disorders, and cancer ${ }^{(16)}$. Several experiments have demonstrated the positive effects of $n-3$ PUFA in preventing the complication of chemotherapy, especially cyclosporine use ${ }^{(17)}$.

The phospholipids of immune cells taken from rodents maintained on normal laboratory chow typically contain $15-20 \%$ of fatty acids as arachidonic acid and contain very little of the very long-chain $n-3$ PUFA $^{(18,19)}$. Ingestion of EPA and DHA from fish partially replaces $n-6$ fatty acids (especially arachidonic acid) in cell membranes, especially those of platelets, erythrocytes, neutrophils, monocytes and liver cells, leading to decreased production of PGE2 metabolites, decreased concentrations of thromboxane A2 (a potent

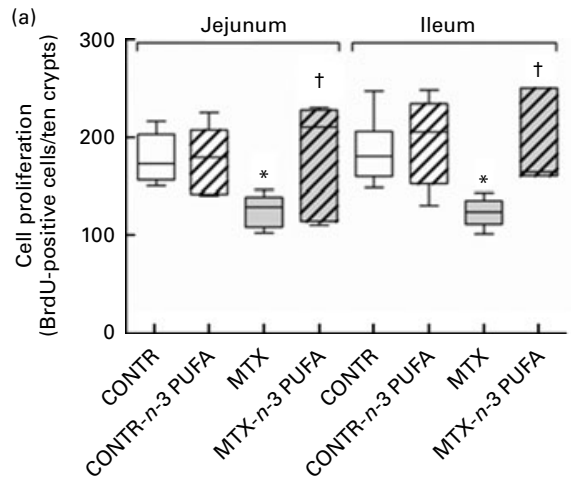

platelet aggregator and vasoconstrictor), decreased formation of leukotriene B4 (an inducer of inflammation and a powerful inducer of leucocyte chemotaxis and adherence), increased concentrations of thromboxane A3 (a weak platelet aggregator and vasoconstrictor), increased concentrations of prostacyclin PGI3 (active vasodilators and inhibitors of platelet aggregation), and increased concentrations of leukotriene B5 (a weak inducer of inflammation and chemotactic agent) ${ }^{(2,20)}$. The recognition that $n-3$ PUFA possess a range of antiinflammatory and immunomodulatory properties has prompted a series of studies investigating their efficacy in animal models of inflammatory bowel disease ${ }^{(21)}$ as well as in clinical trials ${ }^{(22,23)}$. The effect of $n$-3 PUFA during

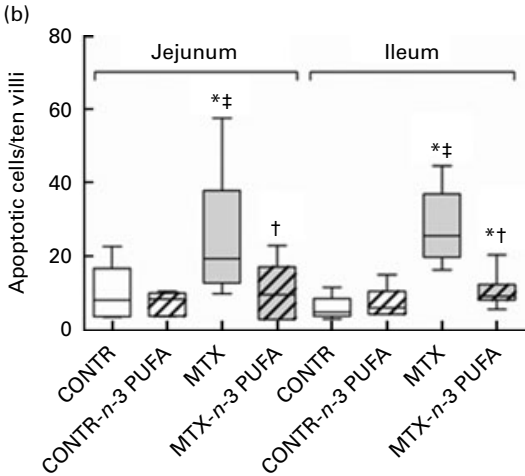

Fig. 6. Effects of methotrexate (MTX) and enteral $n$-3 PUFA on cell (a) proliferation and (b) apoptosis. The number of bromodeoxyuridine (BrdU)-labelled cells in ten well-oriented, longitudinal crypts per section from each rat was determined using light microscopy. Identification of apoptotic cells was performed using immunohistochemistry for caspase-3. The apoptotic index is expressed as the percentage of apoptotic cells per ten villi. Values are means, with their standard errors represented by vertical bars. * Mean values were significantly different for MTX and MTX- $n-3$ PUFA rats from those of the control $($ CONTR) rats $(P<0.05)$. $†$ Mean values were significantly different for MTX-3-PUFA rats from those of MTX rats $(P<0.05)$. $\ddagger$ Mean values were significantly different for MTX and MTX- $n$ - 3 PUFA rats from those of CONTR- $n-3$ PUFA rats $(P<0.05)$. 

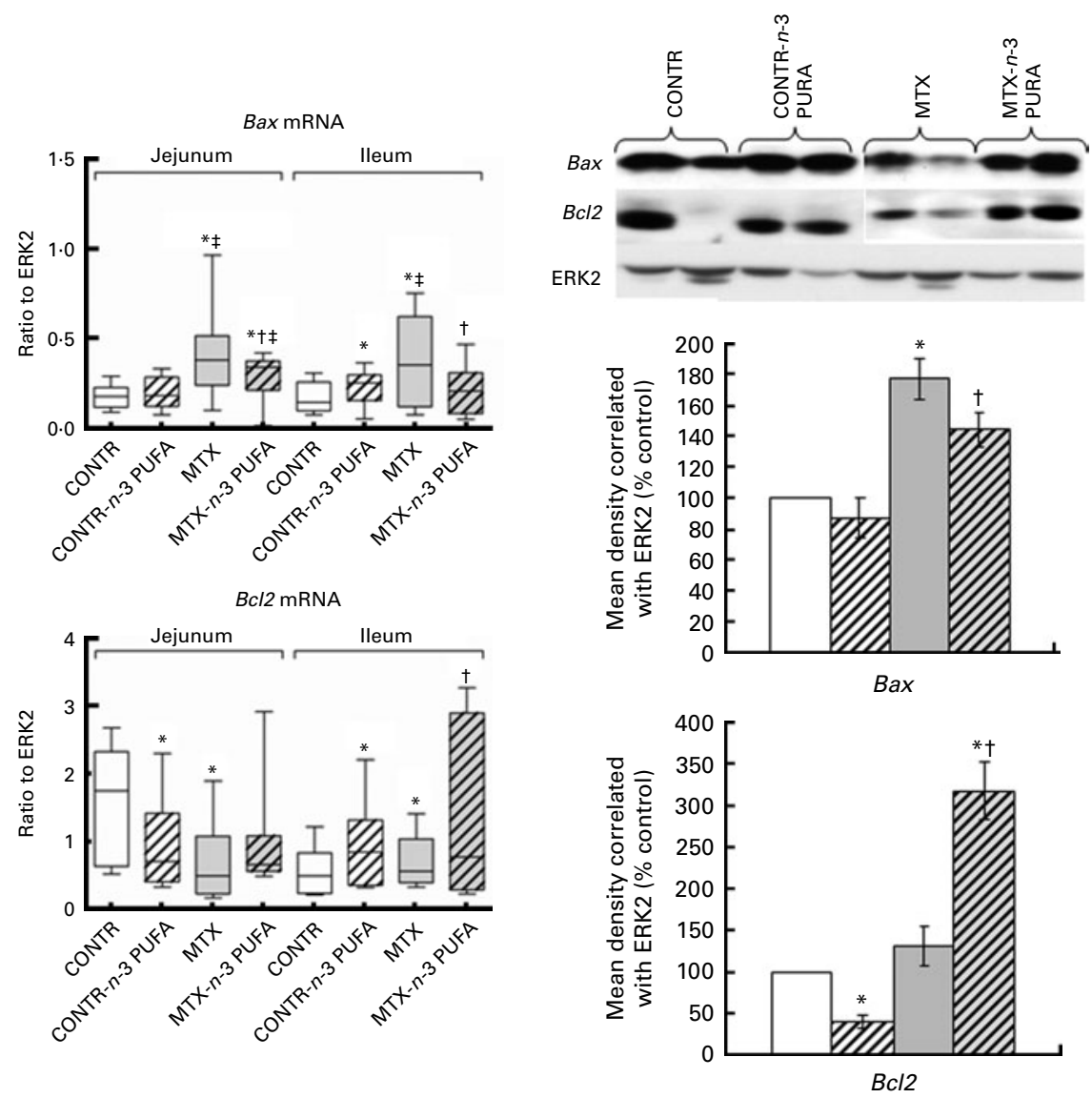

Fig. 7. Effect of $n$-3 PUFA on B-cell lymphoma 2 (Bcl2)-associated X protein (Bax) and Bcl2 mRNA (real-time PCR) and protein (Western blot) levels in the gut mucosa following methotrexate (MTX)-induced intestinal damage. Results are expressed as the ratio of the investigated $B a x$ and $B c / 2$ mRNA to extracellular signal-related kinase (ERK). Values are means, with their standard errors represented by vertical bars. * Mean values were significantly different for methotrexate $(\square)$ and MTX- $n-3$ PUFA $(\mathbb{Z})$ rats from those of the control (CONTR, $\sqsubset)$ rats $(P<0.05)$. $†$ Mean values were significantly different for MTX-3-PUFA rats from those of MTX rats $(P<0.05)$. $\ddagger$ Mean values were significantly different for MTX and MTX- $n-3$ PUFA rats from those of CONTR- $n-3$ PUFA rats $(\mathbb{Z})(P<0.05)$.

chemotherapy-induced intestinal damage has not been previously investigated.

The pathogenesis of chemotherapy-induced gastrointestinal mucositis has been described by Sonis et al. ${ }^{(13)}$, and includes five phases: initiation by chemotherapy; up-regulation and generation of messenger signals; signalling by pro-inflammatory cytokines and amplification of mucosal injury; ulceration of the mucosa; and, finally, healing. The initial stages of inflammation in mucositis include increased pro-inflammatory cytokine levels, which act as a homing marker for inflammatory immune cells in the submucosa. Since anti-inflammatory properties of $n-3$ PUFA have been assessed in a number of disease settings ${ }^{(6-10)}$, we hypothesised in this experiment that $n-3$ fatty acids might reduce the severity of chemotherapyinduced mucositis.

The present study demonstrates that enteral $n-3$ PUFA in the control animals did not change significantly intestinal mucosal parameters. This conclusion is supported by unchanged bowel and mucosal weight, mucosal DNA and protein, villus height and crypt depth.

Consistent with our previous experiments ${ }^{(24,25)}$, the present findings have shown that MTX-induced mucositis results in significant mucosal damage. This conclusion is supported by the observed increase in intestinal injury score compared with the control animals. Histologically, MTX animals exhibited degeneration and shortening of the villus length, severe villous epithelial atrophy, a significant loss of the crypt architecture, signs of crypt remodelling, and polymorphonuclear leucocyte infiltration in the lamina propria. Vacuolisation in surface epithelium cells was minimal. In addition, MTX rats showed intestinal mucosal hypoplasia. This is evident from decreased bowel and mucosal weight, decreased mucosal DNA and protein, and decreased villus height and crypt depth in this model. Decreased body weight in MTX rats may suggest a malnutrition state. Parallel decreases in mucosal DNA and protein indicate that the smaller intestinal mass of MTX animals can be attributed to cellular hypoplasia. The most significant differences were observed in the terminal ileum, while hyperplasia in the proximal jejunum was less prominent. BrdU was used in our experiment to determine an index of crypt cell proliferation. This analogue of thymidine is incorporated into the DNA of proliferating cells during the S-phase of the cell cycle. The present findings suggest that proliferation of crypt cells decreased significantly following MTX administration, which was closely correlated with decreased crypt depth. MTX and its active metabolites 

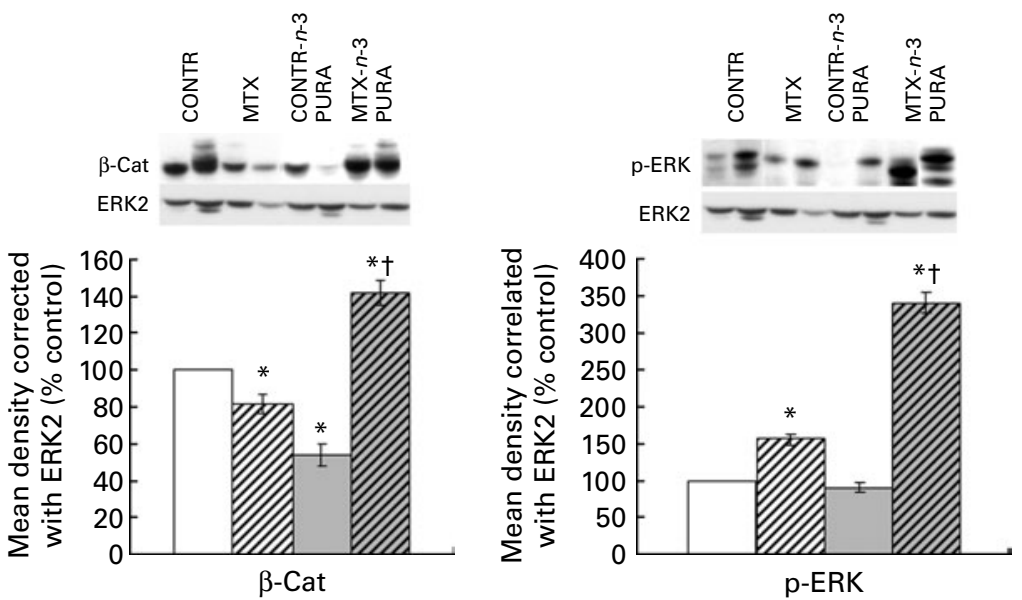

Fig. 8. Effects of methotrexate (MTX, $\square$ ) and enteral $n$-3 PUFA on proliferation ((a) $\beta$-catenin ( $\beta$-cat) and (b) phosphorylated extracellular signal-related kinase $(p-E R K))$-related proteins (Western blot). Results are calculated as the ratio to ERK and are expressed as a percentage of the control animals. Values are means, with their standard errors represented by vertical bars. ${ }^{*}$ Mean values were significantly different for MTX and MTX- $n-3$ PUFA rats $(\mathbb{Z})$ from those of the control (CONTR, $ᄃ)$ rats $(P<0.05)$. † Mean values were significantly different for MTX-3-PUFA rats from those of MTX rats $(P<0.05)$. $飞$, CONTR- $n-3$ PUFA.

block tetrahydrofolate synthesis by binding to the folic acid site on the enzyme dihydrofolate reductase. This action results in the depletion of nucleotide precursors, and the inhibition of DNA, RNA, protein synthesis and cellular proliferation. Decreased cell proliferation rate was accompanied by lower $\beta$-catenin levels. Extensive experimental evidence suggests that Wnt/ $\beta$-catenin signalling plays a central role in maintaining the intestinal stem-cell niche and in regulating differentiation of stem cells within the intestinal epithelium towards either enterocytes or one of three secretory cell lineages ${ }^{(23)}$. Cell loss in the small intestine of MTX-induced mucositis is mainly regulated by programmed cell death. The present results show that the intrinsic pathway, with its regulation by the $\mathrm{Bcl} 2$ family of proteins, was altered by MTX, consistent with changes in cell apoptosis; the mRNA levels of the proapoptotic gene Bax increased, while those of the anti-apoptotic $\mathrm{Bcl} 2$ gene decreased. Changes in protein levels are in agreement with mRNA expression. Bax protein level was significantly up-regulated in MTX-treated rats compared with the control animals. These changes correlated with the enhanced enterocyte apoptosis during MTX-induced mucositis.

In the present study, administration of enteral $n$ - 3 PUFA in MTX rats decreased mucosal inflammation and reversed intestinal mucosal damage. This is evident from the decreased intestinal injury score. Although the mucosa was still severely damaged, MTX- $n-3$ PUFA rats showed the presence of newly formed crypts and regeneration, less significant villous epithelial atrophy and less significant polymorphonuclear leucocyte infiltration in the lamina propria. The mechanisms of this effect are unknown. Further studies are required to determine whether decreased generation of pro-inflammatory cytokines (TNF- $\alpha$, IL-1 $\beta$, IL-6 and IL-8) as well as decreased activation of NF- $\mathrm{BB}$ signalling, decreased leucocyte chemotaxis and decreased T-cell reactivity, which were described in an animal model of inflammatory bowel disease ${ }^{(26)}$, may be responsible for the observed protective effects of $n-3$ PUFA in chemotherapy-induced mucositis. In addition, reduced inflammation was coupled with enhanced intestinal repair.
Enteral $n$-3 PUFA significantly increased overall bowel and mucosal weight, which occurred together with a synergistic increase in mucosal DNA and protein levels, which indicates that the greater intestinal mass is attributed to cellular hyperplasia. n-3 PUFA also caused a significant stimulation of cell proliferation and a concomitant decrease in cell apoptosis compared with MTX-untreated rats, which suggests an activated enterocyte turnover and may be considered as one of the main mechanisms of mucosal hyperplasia in recovering bowel. It has been suggested that incorporation of $n-3$ PUFA into the intestinal mucosa may have altered mucosal function and could have contributed to the regulation of growth and transduction signals such as those involving protein kinase $\mathrm{C}^{(27)}$; however, further investigation is required to ascertain the mechanisms by which $n-3$ PUFA enhance repair in the intestinal mucosa. The transmission of extracellular proliferation and differentiation signals into their intracellular targets is mediated by a signalling cascade culminating in mitogenactivated protein kinase (MAPK). MAPK are serine/ threonine-specific protein kinases that regulate various cellular activities, such as gene expression, mitosis, cell proliferation, differentiation and apoptosis ${ }^{(28)}$. One of the MAPK signalling pathways triggered by cytokines or growth factors is the ERK pathway. In the present study, the observed increase in enterocyte proliferation rate in MTX- $n$ - 3 PUFA animals was accompanied by elevated $\beta$-catenin and phosphorylated ERK protein levels, which suggests activated stem cell activity and a stimulated MAPK signalling pathway. Treatment with $n$-3 PUFA also resulted in decreased cell apoptosis. Bax mRNA levels were down-regulated, while Bcl2 mRNA was up-regulated in $n$-3 PUFA-treated rats compared with MTXnon-treated animals. Thus, the Bax:Bcl2 ratio decreased in MTX- $n-3$ PUFA rats compared with MTX animals, suggesting increased enterocyte survival. These results suggest that n-3 PUFA may inhibit chemotherapy-induced apoptosis by concomitant stimulation of the anti-apoptotic Bcl2 gene and inhibition of the pro-apoptotic Bax gene expression. Both elevated cell proliferation and decreased cell apoptosis may 
be considered as one of the main mechanisms responsible for compensatory hyperplasia and, thus, responsible for increased intestinal cell mass

The mechanisms of the positive effects of PUFA on intestinal mucosal homeostasis are poorly understood. Several experiments have demonstrated that exposure of animals to a PUFA-enriched diet results in an increased PUFA concentration in ileal mucosa. In the present study, we have also shown the stimulating effect of PUFA on intestinal mucosa in the ileum. Since PUFA are absorbed mostly in the proximal intestine, it should be emphasised that the effects in the distal intestine are probably systemic. However, the local effect of small amounts of PUFA that did not get absorbed in the proximal jejunum cannot be excluded. Since changes in serum and tissue levels of NF-кB, TNF, IL- $1 \beta$ and IL-6 have been demonstrated during chemotherapy-induced mucositis ${ }^{(29)}$, further studies are required to evaluate cytokine and key inflammatory protein tissue levels to define trafficking pathways of anti-inflammatory effects of PUFA in this model.

In conclusion, enteral $n-3$ fatty acids reverse intestinal damage and stimulate intestinal recovery following MTXinduced intestinal damage in a rat. Enhanced cell proliferation and inhibited programmed cell death (through the upregulation of $\mathrm{Bcl} 2$ and the down-regulation of $\mathrm{Bax}$ expression) may be responsible for this effect. Enteral $n-3$ fatty acids may be clinically beneficial in preventing intestinal damage and in stimulating intestinal recovery in patients with chemotherapy-induced mucositis.

\section{Acknowledgements}

This study was supported by a research grant from the Israeli Ministry of Health no. 3/6164 from 01/06/10. T. K. and I. S. conceived and participated in the design, experimental work and collection of the data, analysis and interpretation of the results, and drafting and substantial editing of the manuscript. Y. P. and J. B. were responsible for the experimental work and collection of the data, and analysis and interpretation of the results. J. M. conceived and participated in the design, analysis and interpretation of the results. A. G. C. conceived and participated in the design, drafting and substantial editing of the manuscript. The authors declare that there are no conflicts of interest.

\section{References}

1. Simopoulos AP (1999) Essential fatty acids in health and chronic disease. Am J Clin Nutr 70, Suppl., 560S-569S.

2. Weber PC, Fischer S, von Schacky C, et al. (1986) Dietary omega-3 polyunsaturated fatty acids and eicosanoid formation in man. In Health Effects of Polyunsaturated Fatty Acids in Sea Foods, pp. 49-60 [AP Simopoulos, RR Kifer and RE Martin, editors]. Orlando, FL: Academic Press.

3. Ekema G, Falchetti D, Boroni G, et al. (2008) Reversal of severe parenteral nutrition-associated liver disease in an infant with short bowel syndrome using parenteral fish oil (Omega-3 fatty acids). J Pediatr Surg 43, 1191-1195.

4. Eisner F, Jacob P, Frick JS, et al. (2011) Immunonutrition with long-chain fatty acids prevents activation of macrophages in the gut wall. J Gastrointest Surg 15, 853-859.
5. Meydani SN, Lichtenstein AH, Cornwall S, et al. (1993) Immunologic effects of national cholesterol education panel step-2 diets with and without fish-derived $N-3$ fatty acid enrichment. J Clin Invest 92, 105-113.

6. Loeschke K, Ueberschaer B \& Pietsch A (1996) n-3 Fatty acids only delay early relapse of ulcerative colitis in remission. Dig Dis Sci 41, 2087-2094.

7. Aslan A \& Triadafilopoulos G (1992) Fish oil fatty acid supplementation in active ulcerative colitis: a double-blind, placebo-controlled, crossover study. Am J Gastroenterol 87, 432-437.

8. Kremer JM, Lawrence DA, Petrillo GF, et al. (1995) Effects of high-dose fish oil on rheumatoid arthritis after stopping nonsteroidal antiinflammatory drugs: clinical and immune correlates. Arthritis Rheum 38, 1107-1114.

9. Geusens P, Wouters C, Nijs J, et al. (1994) Long term effect of omega-3 fatty acid supplementation in active rheumatoid arthritis: a 12 month, double blind, controlled study. Arthritis Rheum 37, 824-829.

10. Schmöcker C, Weylandt KH, Kahlke L, et al. (2007) Omega-3 fatty acids alleviate chemically induced acute hepatitis by suppression of cytokines. Hepatology 45, 864-869.

11. Sonis ST (1993) Complications of cancer and their treatment: oral complications. In Cancer Medicine, 3rd ed., pp. 2381-2388 [JF Holland, E Frei and RC Bast, editors]. Philadelphia: Lea and Febiger.

12. Naidu MU, Ramana GV, Rani PU, et al. (2004) Chemotherapyinduced and/or radiation therapy-induced oral mucositis? Complicating the treatment of cancer. Neoplasia 6, 423-431.

13. Sonis ST, Elting LS, Keefe DMK, et al. (2004) Perspectives on cancer therapy-induced mucosal injury: pathogenesis, measurement, epidemiology, and consequences for patients. Cancer 100, 1995-2025.

14. Kesik V, Uysal B, Kurt B, et al. (2009) Ozone ameliorates methotrexate-induced intestinal injury in rats. Cancer Biol Ther 8, 1623-1628.

15. Dyerberg J, Bang HO \& Hjorne N (1975) Fatty acid composition of the plasma lipids in Greenland Eskimos. Am J Clin Nutr 28, 958-967.

16. Simopoulos AP, Kifer RR, Wykes AA, et al. (1991) Omega 3 fatty acids: research advances and support in the field since June 1985 (worldwide). World Rev Nutr Diet 66, $51-71$.

17. Levy AE \& Alexander JW (1995) Nutritional immunomodulation enhances cardiac allograft survival in rats treated with donorspecific transfusion and CsA. Transplantation 60, 812-815.

18. Calder PC, Yaqoob P, Harvey DJ, et al. (1994) The incorporation of fatty acids by lymphocytes and the effect on fatty acid composition and membrane fluidity. Biochem $J \mathbf{3 0 0}$, 509-518

19. Yaqoob P, Newsholme EA \& Calder PC (1995) Influence of cell culture conditions on diet-induced changes in lymphocyte fatty acid composition. Biochim Biophys Acta 1255, 333-340.

20. Lewis RA, Lee TH \& Austen KF (1986) Effects of omega-3 fatty acids on the generation of products of the 5-lipoxygenase pathway. In Health Effects of Polyunsaturated Fatty Acids in Sea Foods, pp. 227-238 [AP Simopoulos, RR Kifer and RE Martin, editors]. Orlando, FL: Academic Press.

21. Shoda R, Matsueda K, Yamato S, et al. (1995) Therapeutic efficacy of $n-3$ polyunsaturated fatty acid in experimental Crohn's disease. J Gastroenterol 30, Suppl. 80, 98-101.

22. Hillier K, Jewell R, Dorrell L, et al. (1991) Incorporation of fatty acids from fish oil and olive oil into colonic mucosal lipids and effects upon eicosanoid synthesis in inflammatory bowel disease. Gut 32, 1151-1155. 
23. Haegebarth A \& Clevers H (2009) Wnt signaling, Lgr5, and stem cells in the intestine. Am J Pathol 174, 15-21.

24. Sukhotnik I, Mogilner J, Karry R, et al. (2009) Effect of oral glutamine on enterocyte turnover during methotrexateinduced mucositis in a rat. Digestion 79, 5-13.

25. Sukhotnik I, Mogilner JG, Shteinberg D, et al. (2009) Leptin accelerates enterocyte turnover during methotrexateinduced intestinal mucositis in a rat. Cancer Biol Ther 8, 1-7.

26. Calder PC (2009) Fatty acids and immune function: relevance to inflammatory bowel diseases. Int Rev Immunol $\mathbf{2 8}$, $506-534$
27. Calderaro V, Parrillo C, Balestrieri ML, et al. (1994) Docosahexaenoic acid and signaling pathways in rabbit colon. Mol Pharmacol 45, 737-746.

28. Pearson G, Robinson F, Beers Gibson T, et al. (2001) Mitogen-activated protein (MAP) kinase pathways: regulation and physiological functions. Endocr Rev 22, $153-183$.

29. Logan RM, Stringer AM, Bowen JM, et al. (2008) Serum levels of NF-кB and pro-inflammatory cytokines following administration of mucotoxic drugs. Cancer Biol Ther 7, $1139-1145$ 